\title{
Green 公式和代数的遗传性*
}

\author{
张光连 $^{* *}$
}

(清华大学数学科学系, 北京 100084 ; 盐城师范学院, 盐城 224002)

王 帅

(Department of Mathematics, The Johns Hopkins University, Baltimore, MD21205, USA)

摘要 设 $\Lambda$ 是有限域 $k$ 上的有限维代数, 证明了在 $\bmod \Lambda$ 中 Green 公式成立当 且仅当 $\Lambda$ 是遗传代数.

\section{关键词 Green 公式 幂等理想 遗传代数}

\section{1 引言}

设 $k$ 是有限域, 含有 $q$ 个元素, $\Lambda$ 是有限维 $k$ - 代数, 我们记有限维 (有限) 左 $\Lambda$ - 模构成的范畴为 $\bmod \Lambda$. 对于 $M \in \bmod \Lambda,[M]$ 表示 $M$ 的同构类. $P(\Lambda)=$ $\{[M] \mid M \in \bmod \Lambda\}$. 若 $X$ 是有限集, 令 $|X|$ 表示 $X$ 的集合基数. 若 $\alpha \in P(\Lambda)$, 取 定 $V_{\alpha} \in \alpha$, 令 $a_{\alpha}=\left|\operatorname{Aut}_{\Lambda}\left(V_{\alpha}\right)\right|$.

称 $\Lambda$ 是遗传代数，如果 2 阶同调算子 $\operatorname{Ext}_{\Lambda}^{2}(-,-)$ 在 $\bmod \Lambda$ 中总等于 0 , 即 $\operatorname{Ext}_{\Lambda}^{2}(M, N)=0$, 对任意 $M, N \in \bmod \Lambda$.

在文献 [1] 中, Green 给出了以下

定理 1.1 设 $\Lambda$ 是有限域 $k$ 上有限维遗传代数, 则对于 $\bmod \Lambda$ 中任意 4 个模 $V_{\alpha}, V_{\beta}, V_{\alpha^{\prime}}, V_{\beta^{\prime}}$, 成立公式

$$
\begin{aligned}
& a_{\alpha} a_{\beta} a_{\alpha^{\prime}} a_{\beta^{\prime}} \sum_{\lambda \in \mathcal{P}} g_{\alpha \beta}^{\lambda} g_{\alpha^{\prime} \beta^{\prime}}^{\lambda} a_{\lambda}^{-1} \\
= & \sum_{\rho, \sigma, \sigma^{\prime} \tau \in \mathcal{P}} \frac{\left|\operatorname{Ext}^{1}\left(V_{\rho}, V_{\tau}\right)\right|}{\left|\operatorname{Hom}\left(V_{\rho}, V_{\tau}\right)\right|} \cdot g_{\rho \sigma}^{\alpha} g_{\sigma^{\prime} \tau}^{\beta} g_{\rho \sigma^{\prime}}^{\alpha^{\prime}} g_{\sigma \tau}^{\beta^{\prime}} a_{\rho} a_{\sigma} a_{\sigma^{\prime}} a_{\tau},
\end{aligned}
$$


这里 $g_{\alpha \beta}^{\lambda}$ 表示 $V_{\lambda}$ 的子模 $N$ 的个数, 使得 $N \simeq V_{\beta}$ 且 $V_{\lambda} / N \simeq V_{\alpha}$.

详细的证明可参见文献 [1,2].

由此公式出发, 可以构造有限维遗传代数的 Ringel-Hall 代数的余乘和对极, 使其成为 Hopf 代数 (见文献 [2,3]), 进而构造 Drinfeld double 和完整实现可对称化 (广义)Kac-Moody Lie 代数的量子包络代数 ${ }^{[2 \sim 4]}$, 但是 Ringel-Hall 代数对于一般 有限维代数的模范畴依然存在. 一个自然的问题是: 一般有限维代数的模范畴 所导出的 Ringel-Hall 代数是否具有由 Green 公式诱导的 Hopf 代数结构. 本文将 证明, 如果 Green 公式对于一个有限维代数的模范畴成立, 则此有限维代数必是 遗传代数，所以 Green 公式意味着遗传性.

\section{2 预备}

设 $\Lambda$ 是带有单位元的结合环. 如果 $J^{2}=J$, 则 $\Lambda$ 的理想 $J$ 称为幂等理想. 一般地，如果 $M$ 是 $\Lambda / J$ - 模，则自然地可将 $M$ 看成 $\Lambda$ - 模，其中 $J$ 是 $M$ 的零化 子.

引理 2.1 设 $\Lambda$ 是带有单位元的结合环， $J$ 是 $\Lambda$ 的幕等理想， $X, Y \in$ $\bmod \Lambda / J$, 则

(i) $\operatorname{Hom}_{\Lambda}(X, Y)=\operatorname{Hom}_{\Lambda / J}(X, Y), \operatorname{Aut}_{\Lambda}(X)=\operatorname{Aut}_{\Lambda / J}(X)$,

(ii) $\operatorname{Ext}_{\Lambda}^{1}(X, Y)=\operatorname{Ext}_{\Lambda / J}^{1}(X, Y)$.

证 (i) 显然.

(ii) 设 $0 \rightarrow Y \stackrel{\mu}{\rightarrow} E \stackrel{\nu}{\rightarrow} X \rightarrow 0$ 是 $\bmod \Lambda$ 中正合列，如果 $X, Y$ 是 $\Lambda / J$ - 模，即 $J X=J Y=0$, 则由 $J X=0$ 知 $J E \subseteq \mu(Y)$, 从而 $J E=J^{2} E \subseteq J \mu(Y)=\mu(J Y)=$ $\mu(0)=0$, 即 $E$ 是 $\Lambda / J$ - 模.

反过来，若 $E$ 是 $\Lambda / J$ 模，即 $J E=0$, 由于 $\mu$ 是同态单射， $\nu$ 是同态满射， 所以 $J X=0=J Y$, 即 $X$ 和 $Y$ 是 $\Lambda / J$ 模.

由引理 2.1 , 立即知

命题 2.2 设 $\Lambda$ 是有单位元的有限维 $k$-代数， $J$ 是 $\Lambda$ 的幕等理想，则对于 取定的 $\alpha, \beta, \alpha^{\prime}, \beta^{\prime} \in P(\Lambda / J)$, 其 Green 公式在 $\bmod \Lambda$ 中成立当且仅当对应 Green 公式在 $\bmod \Lambda / J$ 中成立.

引理 2.3 设 $\Lambda$ 是一个带单位元的结合环， $J$ 是 $\Lambda$ 的理想， $M$ 是 $\Lambda$ - 模, 则 $\operatorname{Hom}_{\Lambda}(\Lambda / J, M) \simeq\{m \in M \mid J m=0\}$.

证 令映射 $\varphi: \operatorname{Hom}_{\Lambda}(\Lambda / J, M) \rightarrow\{m \in M \mid J m=0\}$, 将 $f$ 映射到 $f(\overline{1})$. 对 任意 $a \in J, a f(\overline{1})=f(a \overline{1})=f(\overline{0})=0$, 当然 $f$ 也由 $f(\overline{1})$ 惟一确定，所以映射 $\varphi$ 是 合理定义的, 显然 $\varphi$ 是双射.

引理 2.4 设 $\Lambda$ 是有限维 $k$ - 代数, $X$ 和 $Y$ 是不可分解模, 但 $M$ 是可分解 $\Lambda$ - 模，如果 $\operatorname{Hom}_{\Lambda}(X, Y)=0$, 则以下任意形式的正合序列

$$
0 \rightarrow X \stackrel{f}{\rightarrow} M \stackrel{g}{\rightarrow} Y \rightarrow 0
$$

都是分裂的. 
证 设 $M=M_{1} \oplus M_{2}, M_{1}$ 和 $M_{2}$ 非零, 设自然投影 $\pi_{i}: M \rightarrow M_{i}(i=1,2)$, 将 函子 $\operatorname{Hom}_{\Lambda}(X,-)$ 应用于正合序列，由于 $\operatorname{Hom}_{\Lambda}(X, Y)=0$, 得到 $\operatorname{Hom}_{\Lambda}(X, X) \simeq$ $\operatorname{Hom}_{\Lambda}(X, M)$, 那么存在 $t \in \operatorname{Hom}_{\Lambda}(X, X)$, 使得 $f t=\pi_{1} f$, 则 $f t=\pi_{1} f=\pi_{1}^{2} f=$ $\pi_{1} f t=f t^{2}$. 由于 $f$ 是单射, 得到 $t=t^{2}$, 又由于 $\operatorname{End}_{\Lambda}(X)$ 是局部环, 所以只可能 $t=0$ 或者 1 , 则 $\pi_{1} f=0$ 或者 $f$. 同理 $\pi_{2} f=0$ 或者 $f$, 当 $\pi_{1} f=0$ 时 $\pi_{2} f=f$, 当 $\pi_{1} f=f$ 时 $\pi_{2} f=0$, 即有原正合序列可裂.

引理 2.5 [1] 设 $M$ 是 $\Lambda$ - 模, 若 $M=M_{1} \oplus M_{2}$, 且 $M_{1}$ 与 $M_{2}$ 没有相互同构 的直和项, 则

$$
\begin{gathered}
a_{M}=a_{M_{1}} a_{M_{2}} q^{\operatorname{dim}_{k} \operatorname{Hom}_{\Lambda}\left(M_{1}, M_{2}\right)+\operatorname{dim}_{k} \operatorname{Hom}_{\Lambda}\left(M_{2}, M_{1}\right)}, \\
g_{M_{1}, M_{2}}^{M_{1} \oplus M_{2}}=q^{\operatorname{dim}_{k} \operatorname{Hom}_{\Lambda}\left(M_{2}, M_{1}\right)} .
\end{gathered}
$$

引理 2.6 设 $X, Y$ 是 $\Lambda$ - 模, $Y$ 不可分解, 满足 $\operatorname{Hom}_{\Lambda}(X, Y)=0$, 如果 $0 \rightarrow X \stackrel{\mu}{\rightarrow} E \stackrel{g}{\rightarrow} Y \rightarrow 0$ 是 $\bmod \Lambda$ 中的正合列, $E$ 有分解式 $E=E_{1} \oplus E_{2}$, 则 $X \simeq E_{1} \oplus M, M$ 为 $E_{2}$ 的子模，或者 $X \simeq N \oplus E_{2}, N$ 为 $E_{1}$ 的子模,

证 作用 $\operatorname{Hom}_{\Lambda}(-, Y)$ 于正合列, 由于 $\operatorname{Hom}_{\Lambda}(X, Y)=0$ 得到 $g^{*}: \operatorname{Hom}_{\Lambda}(Y, Y)$ $\rightarrow \operatorname{Hom}_{\Lambda}(E, Y)$ 是同构, 令 $\pi_{i}: E \rightarrow E_{i} \hookrightarrow E$ 是关于分解式 $E=E_{1} \oplus E_{2}$ 的自然投影 $(i=1,2)$, 则 $g \pi_{1} \in \operatorname{Hom}_{\Lambda}(E, Y)$, 所以存在 $f \in \operatorname{Hom}_{\Lambda}(Y, Y)$, 使得 $f g=g \pi_{1}$, 那么 $f g=g \pi_{1}=g \pi_{1}^{2}=f g \pi_{1}=f^{2} g$, 所以 $\left(f-f^{2}\right) g=0$, 由于 $g$ 是 满射, 所以 $f-f^{2}=0$, 即 $f^{2}=f$. 又因为 $\operatorname{End}_{\Lambda} Y$ 是局部环, 所以 $f=0$ 或 者 $f=1$, 也即 $g \pi_{1}=0$ 或者 $g \pi_{1}=g$. 如果 $g \pi_{1}=0$, 那么 $E_{1} \subset \operatorname{Ker} g$, 所以 $\operatorname{Ker} g=\operatorname{Ker} g \cap E=\operatorname{Ker} g \cap\left(E_{1} \oplus E_{2}\right)=E_{1} \oplus \operatorname{Ker} g \cap E_{2}$, 则 $X \simeq \operatorname{Ker} g=E_{1} \oplus M, M$ 是 $E_{2}$ 的子模. 如果 $g \pi_{1}=g$, 由于 $g=g \pi_{1}+g \pi_{2}$, 则 $g \pi_{2}=0$, 同理可得 $X \simeq N \oplus E_{2}$, $N$ 是 $E_{1}$ 的子模.

引理 2.7 设 $A$ 是有限域 $k$ 上的有限维局部代数, 其极大理想 $J \neq 0$, 则存 在 $\left\{\sigma_{1}, \sigma_{2}, \cdots, \sigma_{s}\right\}$ 为 $J / J^{2}$ 作为左 $A / J$ - 向量空间的一个基, 令 $r_{i} \in p^{-1}\left(\sigma_{i}\right)$, 这里 $p: J \rightarrow J / J^{2}$ 为自然投影，满足

(i) 任意 $x \in J$ 可表示为 $x=\sum a_{i_{1}, i_{2}, \cdots, i_{k}} r_{i_{1}} \cdots r_{i_{k}}$, 其中非零的 $a_{i_{1}, i_{2}, \cdots, i_{k}}$ 为 $A$ 中的可逆元.

(ii) 若 $a \in A$ 是可逆元, 则对任意 $1 \leqslant i \leqslant s, r_{i} a=a^{\prime} r_{i}+\sum_{k \neq i} \sum_{j=1}^{s} b_{j k} r_{j} r_{k}$, 这里 $a^{\prime}, b_{j k} \in A$,

证 (i) 由 Nakayama 引理, 有表达式 $x=\sum_{i} b_{i} r_{i}, b_{i} \in A$, 由于 $A$ 是局部环, 所以 $b_{i}$ 是 $A$ 中的可逆元或 $b_{i} \in J$, 对于非零的 $b_{i} \in J$, 按以上表达式展开, 由根 $J$ 的幕零性知 (i) 成立.

(ii) 由于 $r_{i} \in J$, 在 $A$ 中可表为 $r_{i} a=\sum a_{k} r_{k}$, 设 ( ) $: A \rightarrow A / J$ 是自然同态, 而 $p: J \rightarrow J / J^{2}$ 是左右 $A / J$ - 模同态, 所以在 $J / J^{2}$ 中有 $(*) \sigma_{i} \bar{a}=\sum \overline{a_{k}} \sigma_{k}$.

我们要证明, 存在 $a^{\prime \prime} \in A$ 可逆元, 使得 $\overline{a^{\prime \prime}} \sigma_{i}=\sum \overline{a_{k}} \sigma_{k}$.

事实上, 对于任意非零 $\bar{a} \in A / J, \bar{a}$ 对 $J / J^{2}$ 的右乘引起 $J / J^{2}$ 作为左 $A / J$ - 
向量空间的一个线性变换, 而 $A / J$ 为有限域, $\bar{a}^{|A / J|-1}=1$, 所以 $\bar{a}$ 的右乘引 起的线性变换可以对角化, 对 $A / J$ 中所有非零元 $\bar{a}$, 在 $J / J^{2}$ 上的右乘变换是 一方相互交换的线性变换，因此可找到 $J / J^{2}$ 作为左 $A / J$ - 向量空间的一个基， 不妨设为 $\left\{\sigma_{1}, \cdots, \sigma_{s}\right\}$, 使得所有非零元 $\bar{a}$ 右乘引起的线性变换可同时对角化, 因而存在非零 $\bar{a}^{\prime \prime} \in A / J$, 使得 $\sigma_{i} \bar{a}=\bar{a}^{\prime \prime} \sigma_{i}$, 对 $1 \leqslant i \leqslant s$. 与 $(*)$ 式比较知, $\bar{a}_{i}=\overline{a^{\prime \prime}}, \overline{a_{k}}=0$, 对 $k \neq i$, 则 $a_{i}=a^{\prime \prime}+m, m \in J, a_{k} \in J$, 对 $k \neq i$ 令 $a^{\prime}=a_{i}$, 则有 $r_{i} a=a^{\prime} r_{i}+\sum_{k \neq i} \sum_{j=1}^{s} b_{k j} r_{j} r_{k}$.

\section{3 公式的逆定理}

定理 3.1 设 $\Lambda$ 为有限域 $k$ 上有单位元的有限维基代数, 且 $1=e_{1}+e_{2}+$ $\cdots+e_{n}$ 是单位元关于正交本原幂等元的分解式，如果存在 $i$, 使得其主不可分解 模 $\Lambda e_{i}$ 的自同态环 $\operatorname{End}_{\Lambda}\left(\Lambda e_{i}\right)$ 为一个不是域的局部环，则在 $\bmod \Lambda$ 中 Green 公式 不能普遍成立.

证 设 $R=\operatorname{rad} \Lambda$, 则 $\bar{\Lambda} \cong D_{1} \times D_{2} \times \cdots \times D_{n}$, 这里 $D_{i}=\operatorname{End}_{\Lambda}\left(S_{i}\right), S_{i}=$ $\Lambda e_{i} / R e_{i}$ 都是单模. 而 $S_{i}$ 也可看成正则 $D_{i}$ - 模, 且有 $\bar{\Lambda} \bar{e}_{i} \cong D_{i}$. 令 $V=R / R^{2}$, $V_{j i}=e_{j} \Lambda e_{i}$ 是左 $D_{j}$ 模, 且 $V_{j i} \cong t_{j i} S_{j}$. 选取 $V_{j i}$ 的一个由 $t_{j i}$ 个元素组成的生 成元系, 并把它们看做格式 $S(\Lambda)$ 中由 $i$ 到 $j$ 的箭 ( $t_{j i}$ 个). 设 $v_{\sigma}$ 是对应着箭 $\sigma: i \rightarrow j$ 的生成元, 而 $r_{\sigma}$ 是它在 $R_{j i}=e_{j} R e_{i}$ 中的一个原像, 则 $\left\{v_{\sigma}\right\}$ 组成模 $V$ 的一个生成元系, 进而由 Nakayama 引理, $\left\{r_{\sigma}\right\}$ 为 $R$ 作为左理想的生成元系, 且对于任意 $x \in e_{j} R e_{i}$ 有 $x=\sum a_{\sigma_{k} \cdots \sigma_{1}} r_{\sigma_{k}} \cdots r_{\sigma_{1}}$, 其中 $\Sigma$ 是对一切从 $i$ 到 $j$ 的路 求和, $a_{\sigma_{k} \cdots \sigma_{1}} \in e_{j} \Lambda e_{j}$ 为可逆元.

情形 1 令 $A^{\prime}=\operatorname{End}_{\Lambda}\left(\Lambda e_{1}\right)$, 且 $t_{11} \neq 0$, 则存在 $r_{\sigma} \neq 0$ 且 $r_{\sigma} \in e_{1} \cdot R \cdot e_{1}$, $\sigma: 1 \rightarrow 1$, 进而幕零元 $r_{\sigma} \notin \sum_{i \neq 1} \Lambda e_{i} \Lambda$. 否则, 如果 $r_{\sigma}=\sum_{i \neq 1} a_{i} e_{i} b_{i}, a_{i}, b_{i} \in$ $\Lambda$, 则 $r_{\sigma}=e_{1} r_{\sigma} e_{1}=\sum_{i \neq 1}\left(e_{1} a_{1} e_{i}\right)\left(e_{i} b_{i} e_{1}\right) \in R^{2}$, 与 $0 \neq v_{\sigma}=\bar{r}_{\sigma}$ 矛盾, 故 $A^{\prime} / A^{\prime} \cap \sum_{i \neq 1} \Lambda e_{i} \Lambda \simeq \Lambda / \sum_{i \neq 1} \Lambda e_{i} \Lambda$ 为非域的局部环. 令 $A=\Lambda / \sum_{i \neq 1} \Lambda e_{i} \Lambda, A$ 是非域的局部环, 其极大理想 $J \neq 0 . A / J$ 是惟一单 $A$ - 模 (同构意义下). 设 $\operatorname{dim}_{A / J}\left(J / J^{2}\right)=s$. 取 $\left\{\sigma_{1}, \cdots, \sigma_{s}\right\}$ 和 $\left\{r_{1}, \cdots, r_{s}\right\}$ 的意义同引理 2.7. $\mathfrak{a}$ 为由 $r_{2}, \cdots, r_{s}$ 生成的理想, 则 $A / \mathfrak{a}$ 为 $A$ - 模, 令 $n$ 为使得 $r_{1}^{n-1} \notin \mathfrak{a}$ 的最大正整数, $n \geqslant 2$. 记 $M=A / \mathfrak{a}, N=A / J, \alpha=\beta^{\prime}=[M], \beta=\alpha^{\prime}=[N]$. 如果 $\lambda \in P$, 使得 $g_{\alpha \beta}^{\lambda} g_{\alpha^{\prime} \beta^{\prime}}^{\lambda} \neq 0$, 则断言对任意 $E \in \lambda$ 有 $E \simeq M \oplus N$. 因为这时有正合列

$$
\begin{aligned}
& 0 \longrightarrow A / J \stackrel{f_{1}}{\longrightarrow} E \stackrel{g_{1}}{\longrightarrow} A / \mathfrak{a} \longrightarrow 0, \\
& 0 \longrightarrow A / \mathfrak{a} \stackrel{f_{2}}{\longrightarrow} E \stackrel{g_{2}}{\longrightarrow} A / J \longrightarrow 0 .
\end{aligned}
$$

由 (1) 式知, 如果 $\mathfrak{a} E=0$, 则 (1) 式可看做 $A / \mathfrak{a}-$ 模的正合列, 从而可裂, 即 有 $E \simeq M \oplus N$.

如果 (1) 和 (2) 式都不可裂, 则 $\mathfrak{a} E \neq 0, g_{1}(\mathfrak{a} E)=\mathfrak{a} g_{1}(E)=0$. 所以 $\mathfrak{a} E \subset$ $\operatorname{Ker} g_{1}=\operatorname{Im} f_{1}$, 而 $\operatorname{Im} f_{1}$ 是单 $A$ - 模, 所以这时 $\mathfrak{a} E=\operatorname{Im} f_{1}, J \mathfrak{a} E=0$. 
因为 (1) 式不可裂, 所以 $g_{2} f_{1} \in \operatorname{Hom}_{A}(A / J, A / J)$ 不是同构, 故 $g_{2} f_{1}=0$. 存在 $\mu: A / J \rightarrow A / \mathfrak{a}$ 和 $\nu: A / \mathfrak{a} \rightarrow A / J$, 使得下图可换:

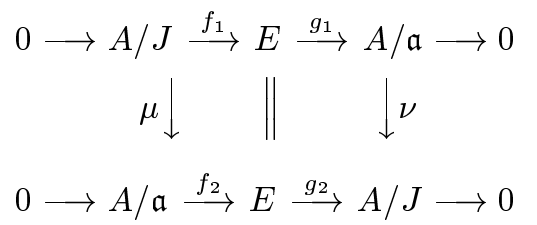

则 $g_{2}(J E)=J g_{2}(E)=0, J E \subset \operatorname{Ker} g_{2}=\operatorname{Im} f_{2}$ ，从而 $\mathfrak{a} J E=0$.

因为 $J \supsetneq \mathfrak{a}, \mu$ 是非同构的单射, 所以 $\mu(1+J)=a r_{1}^{i}+\mathfrak{a}$, 其中 $a \in A$ 可逆, $1 \leqslant i \leqslant n-1$, 由引理 2.3 知 $J\left(a r_{1}^{i}\right) \subset \mathfrak{a}$, 则 $r_{1}\left(a r_{1}^{i}\right) \in \mathfrak{a}$. 由引理 2.7 知 $r_{1}^{i+1} \in \mathfrak{a}$, 故 $i=n-1$.

不妨设 $a=1$ (可调整 $f_{1}$ 为 $f_{1}^{\prime}$, 使得 $f_{1}^{\prime}(1+J)=a^{-1} f_{1}(1+J)$ ). 令 $f_{1}(1+J)=$ $e_{1} \in E, f_{2}(1+\mathfrak{a})=e_{2} \in E$, 由 $f_{1}=f_{2} \mu$ 知

$$
e_{1}=r_{1}^{n-1} e_{2} .
$$

由于 $\nu$ 是满射, 可令 $\nu(1+\mathfrak{a})=b+J$, 其中 $b \in A$ 是可逆元.

再令 $e, \theta \in E$, 使得 $g_{1}(e)=1+\mathfrak{a}, g_{2}(\theta)=1+J$. 由 $g_{2}=\nu g_{1}$ 可得出 $g_{2}(e)=$ $b+J, g_{1}(\theta)=c+\mathfrak{a}$, 其中 $c$ 是 $A$ 中可逆元.

由 $g_{1}\left(e-c^{-1} \theta\right)=0$, 知

$$
e-c^{-1} \theta \in \operatorname{Im} f_{1}=\mathfrak{a} E .
$$

由于正合列 (1) 不可裂, $A / \mathfrak{a}$ 不可分解, 所以 $g_{1} f_{2} \in \operatorname{Hom}_{A}(A / \mathfrak{a}, A / \mathfrak{a})$ 不是同构, 故是幕零映射, 因此 $g_{1} f_{2}(1+\mathfrak{a})=g_{1}\left(e_{2}\right)=d r_{1}^{i}+\mathfrak{a}, i \geqslant 1, d$ 是可逆元.

但 $g_{1}\left(d r_{1}^{i} e\right)=d r_{1}^{i}+\mathfrak{a}$, 所以 $e_{2}=d r_{1}^{i} e+x, x \in \operatorname{Im} f_{1}$. 令 $x=p e_{1}, p \in A$ 是可逆 元, 故 $e_{2}=d r_{1}^{i} e+p e_{1}$, 由 (3) 式得 $e_{2}=d r_{1}^{i} e+p r_{1}^{n-1} e_{2}$, 由于 $1-p r_{1}^{n-1}$ 是可逆元, 故 $e_{2}=d^{\prime} r_{1}^{i} e, d^{\prime}$ 是可逆元, $f_{2}\left(r_{1}^{n-1}+\mathfrak{a}\right)=r_{1}^{n-1} e_{2}=r_{1}^{n-1} d^{\prime} r_{1}^{i} e$. 因为 $e_{2} \in \operatorname{Im} f_{2}$, 故 $r_{1}^{i} e=\left(d^{\prime}\right)^{-1} e_{2} \in \operatorname{Im} f_{2}$, 由引理 2.7 得

$$
f_{2}\left(r_{1}^{n-1}+\mathfrak{a}\right)=d^{\prime \prime} r_{1}^{n+i-1} e,
$$

其中 $d^{\prime \prime}$ 是可逆元. 我们要证明 $r_{1}^{n} e=0$.

由于 $r_{1}^{n} \in \mathfrak{a}$, 可设 $r_{1}^{n}=b_{2} r_{2} c_{2}+\cdots+b_{s} r_{s} c_{s} \in \mathfrak{a}$, 因为 $n \geqslant 2, r_{1}^{n} \in J^{2}$, 所 以在 $A / J$ - 向量空间 $J / J^{2}$ 中, $0=\bar{b}_{2} \sigma_{2} \bar{c}_{2}+\cdots+\bar{b}_{s} \sigma_{s} \bar{c}_{s}$, 由于 $\left\{\sigma_{1}, \sigma_{2}, \cdots, \sigma_{s}\right\}$ 是 左 $A / J$ - 向量空间 $J / J^{2}$ 的基，所以由引理 2.7 的证明知对于每个 $i$ 都有 $b_{i} \in J$ 或 $c_{i} \in J$, 由于 $J \mathfrak{a} E=0, \mathfrak{a} J E=0$, 所以 $b_{2} r_{2} c_{2} e=\cdots=b_{s} r_{s} c_{s} e=0, r_{1}^{n} e=0$, 而 $n+i-1 \geqslant n$, 由 (5) 式知 $f_{2}\left(r_{1}^{n-1}+\mathfrak{a}\right)=0$, 但 $f_{2}$ 是单射, 得出 $r_{1}^{n-1} \in \mathfrak{a}$, 矛盾, 故 $E \simeq M \oplus N$.

在 $\bmod A$ 中, 由于 $M, N$ 是互不同构的不可分解模, 且 $\operatorname{End}_{A}(A / J)$ 为域, 故由引理 2.5 知 Green 公式的左端项是 


$$
\begin{aligned}
a_{\alpha} a_{\beta} a_{\alpha^{\prime}} a_{\beta^{\prime}} \sum_{\lambda \in \mathcal{P}} g_{\alpha \beta}^{\lambda} g_{\alpha^{\prime} \beta^{\prime}}^{\lambda} a_{\lambda}^{-1}= & a_{M}^{2} a_{N}^{2} g_{M, N}^{M \oplus N} g_{N, M}^{M \oplus N} a_{M \oplus N}^{-1} \\
= & a_{M}^{2} a_{N}^{2} q^{\operatorname{dim}_{k} \operatorname{Hom}_{A}(M, N)+\operatorname{dim}_{k} \operatorname{Hom}_{A}(N, M)} \\
& \times a_{M}^{-1} a_{N}^{-1} q^{-\left(\operatorname{dim}_{k} \operatorname{Hom}_{A}(M, N)+\operatorname{dim}_{k} \operatorname{Hom}_{A}(N, M)\right)} \\
= & a_{M} a_{N}=a_{\alpha} a_{\beta} .
\end{aligned}
$$

令 $F=J / \mathfrak{a}$, 存在正合列 $0 \rightarrow J / \mathfrak{a} \rightarrow A / \mathfrak{a} \rightarrow A / J \rightarrow 0$, 即 $0 \rightarrow F \rightarrow M \rightarrow N \rightarrow 0$, 又易证 $0 \rightarrow N \rightarrow M \rightarrow F \rightarrow 0$ 正合, 而 Green 公式的右端每一项都是正的, 所 以 Green 公式右端的值大于或等于

$$
g_{0 \alpha}^{\alpha} g_{\beta 0}^{\beta} g_{0 \beta}^{\beta} g_{\alpha 0}^{\alpha} a_{\alpha} a_{\beta}+\frac{\left|\operatorname{Ext}_{A}^{1}(N, N)\right|}{\left|\operatorname{Hom}_{A}(N, N)\right|} g_{N F}^{M} g_{0 N}^{N} g_{N 0}^{N} g_{F N}^{M} a_{N}^{2} a_{F},
$$

此值严格大于 $a_{\alpha} a_{\beta}$, 所以 Green 公式在 $\bmod A$ 中不能普遍地成立, 由命题 2.2 知, Green 公式在 $\bmod \Lambda$ 中不能普遍成立.

情形 2 如果使得 $\operatorname{End}_{\Lambda}\left(\Lambda e_{i}\right)$ 为非域的局部环的 $i$ 都有 $t_{i i}=0$, 则 $e_{i} R e_{i}$ 中 每一个元都可表示为 $\sum a_{\sigma_{k} \cdots \sigma_{1}} r_{\sigma_{k}} \cdots r_{\sigma_{1}}$, 其中 $\sigma_{k} \cdots \sigma_{1}$ 为从 $i$ 到 $i$ 的路, $a_{\sigma_{k} \cdots \sigma_{1}}$ 为 $e_{i} \Lambda e_{i}$ 中的可逆元.

设 $0 \neq r_{\sigma_{k}} \cdots r_{\sigma_{1}}$ 为所有上述 $\operatorname{End}_{\Lambda}\left(\Lambda e_{i}\right)$ 中元素的表达式中长度最小的圈 $\sigma_{k} \cdots \sigma_{1}$ 所对应的元素, 且 $\sigma_{i}: j_{i-1} \rightarrow j_{i}$, 则 $j_{0}=j_{k}, j_{1}, \cdots, j_{k}$ 互不相同, 不妨设 $j_{0}=1, j_{1}=2, \cdots, j_{k-1}=k$, 那么代数 $\Lambda^{\prime}=\Lambda / \sum_{i \notin\{1,2, \cdots, k\}} \Lambda e_{i} \Lambda$ 的格式 $S\left(\Lambda^{\prime}\right)$ 为 $S(\Lambda)$ 删去顶点 $k+1, \cdots, n$ 以及以此为端点的箭所得到的真子图, 且 $S\left(\Lambda^{\prime}\right)$ 有定 向圈.

不妨设 $p=\sigma_{q} \cdots \sigma_{1}$ 为 $\Lambda^{\prime}$ 的代数关系的直和项中长度最小的一条路, $\sigma_{1}$ : $j_{0} \rightarrow j_{1}, \cdots, \sigma_{q}: j_{q-1} \rightarrow j_{q}$. 令 $r_{p}=r_{\sigma_{q}} \cdots r_{\sigma_{1}},|p|$ 表示路 $\sigma_{q} \cdots \sigma_{1}$ 的长度 $q$. 令 $S=\left\{j_{0}, \cdots, j_{q}\right\}, B=\Lambda^{\prime} / \sum_{i \notin S} \Lambda^{\prime} e_{i} \Lambda^{\prime}$.

令 $M$ 为合成因子依次为 $S_{j_{q-1}}, \cdots, S_{j_{0}}$ 经过路 $\sigma_{q-1} \cdots \sigma_{1}$ 扩张而成的模, $N$ 为 $S_{j_{q}}, M^{\prime}$ 为 $S_{j_{0}}, N^{\prime}$ 为合成因子依次为 $S_{j_{q}}, \cdots, S_{j_{1}}$ 经过路 $\sigma_{q} \cdots \sigma_{2}$ 扩张而成的 模.

由于 $S\left(\Lambda^{\prime}\right)$ 中没有圈, 所以由引理 2.4 知 $M, N, M^{\prime}, N^{\prime}$ 不可分解.

令 $\alpha=[M], \beta=[N], \alpha^{\prime}=\left[M^{\prime}\right], \beta^{\prime}=\left[N^{\prime}\right] \in P(B) \subseteq P\left(\Lambda^{\prime}\right) \subseteq P(\Lambda)$, 则可断言 $g_{\alpha \beta}^{\lambda} g_{\alpha^{\prime} \beta^{\prime}}^{\lambda}=0(\forall \lambda \in P(\Lambda))$.

否则, 如果存在 $\lambda \in P(\Lambda)$, 使得 $g_{\alpha \beta}^{\lambda} g_{\alpha^{\prime} \beta^{\prime}}^{\lambda} \neq 0$, 则由引理 2.1 知 $\lambda \in P(B)$, 设 $E \in \lambda$, 有正合序列

$$
\begin{gathered}
0 \rightarrow N \rightarrow E \rightarrow M \rightarrow 0, \\
0 \rightarrow N^{\prime} \rightarrow E \rightarrow M^{\prime} \rightarrow 0,
\end{gathered}
$$

注意 $S(B)$ 中没有圈, 则 $S_{j_{0}} \nsucceq S_{j_{1}}, S_{j_{q-1}} \nsucceq S_{j_{q}}$, 从而 $\operatorname{Hom}_{B}(N, M)=\operatorname{Hom}_{B}\left(N^{\prime}, M^{\prime}\right)$ $=0$. 
若 $E$ 可分解, 则由引理 2.4 知序列 (6) 和 (7) 均可裂, 从而 $M \oplus N \cong M^{\prime} \oplus N^{\prime}$. 由于 $q \geqslant 2$, 故 $M \nsucceq M^{\prime}$, 又 $j_{0} \neq j_{1}$, 故 $\operatorname{top}(M) \nsucceq \operatorname{top}\left(N^{\prime}\right)$, 从而 $M \nsucceq N^{\prime}$, 此与 $M \oplus N \cong M^{\prime} \oplus N^{\prime}$ 矛盾.

若 $E$ 不可分解, 则由序列 (6) 和 (7) 及 $M, N^{\prime}$ 的构造知, $E$ 必为合成因子 依次为 $S_{j_{q}}, \cdots, S_{j_{1}}, S_{j_{0}}$ 经过路 $\sigma_{q} \cdots \sigma_{1}$ 扩张而成的模. 但由 $p$ 的选择知，存在 $a_{p^{\prime}} \in e_{j} \Lambda^{\prime} e_{j}$ 为可逆元, $p^{\prime}$ 为从 $i$ 到 $j$ 的路, $\left|p^{\prime}\right| \geqslant|p|$, 使得 $r_{p}+\sum_{p^{\prime} \neq p} a_{p^{\prime}} r_{p^{\prime}}=0$ 在代数 $\Lambda^{\prime}$ 中成立, 由 $E$ 的构造知 $r_{p} \cdot E \neq 0, r_{p^{\prime}} E=0$, 故 $E$ 不是 $B$ - 模, 矛盾.

即 Green 公式左端项为 0 .

下证右端项不为 0 , 从而 Green 公式不成立. 为此只需证明右端存在一个非 零项即可.

令 $F=\operatorname{Ker} \varphi\left(\varphi: M \rightarrow M^{\prime}\right)$, 则有正合列 $0 \rightarrow F \hookrightarrow M \rightarrow M^{\prime} \rightarrow 0$. 不妨设 $N$ 为 $N^{\prime}$ 的子模, 则又有正合列 $0 \rightarrow N \hookrightarrow N^{\prime} \rightarrow F \rightarrow 0$.

在右端令 $\rho=\left[M^{\prime}\right], \sigma=[F], \sigma^{\prime}=0, \tau=[N]$, 则该项非零, 故 Green 公式在 $\bmod \Lambda$ 中不能普遍成立.

定理 3.2 设 $\Lambda$ 是有限域 $k$ 上有限维基代数, $1=e_{1}+\cdots+e_{n}$ 是其单位元 关于正交本原幂等元的分解式, 如果对任意 $i, \operatorname{End}_{\Lambda}\left(\Lambda e_{i}\right)$ 是域, 则 Green 公式在 $\bmod \Lambda$ 中普遍成立的充分必要条件是 $\Lambda$ 为遗传代数.

证 假定 $\Lambda$ 不是遗传代数, 则至少存在一个单模, 不妨假定对应于 $e_{1}$ 的单 模为 $S_{1}$, 其投射维数 $\geqslant 2$, 设 $S_{1}$ 的极小投射预解式为

$$
\cdots \rightarrow P_{3} \stackrel{d_{2}}{\rightarrow} P_{2} \stackrel{d_{1}}{\rightarrow} P_{1} \stackrel{d_{0}}{\rightarrow} S_{1} \rightarrow 0,
$$

从而

$$
0 \rightarrow \operatorname{Kerd}_{1} \rightarrow P_{2} \stackrel{d_{1}}{\rightarrow} P_{1} \stackrel{d_{0}}{\rightarrow} S_{1} \rightarrow 0,
$$

且 $\operatorname{Ker} d_{1} \neq 0, P_{1}$ 是 $S_{1}$ 的投射盖，故不可分解， $\operatorname{Ker} d_{0}$ 不是投射模， $P_{2}$ 是 $\operatorname{Ker} d_{0}$ 的投射盖. 令 $P_{2}=P_{21} \oplus \cdots \oplus P_{2 t}$ 为 $P_{2}$ 分解成不可分解模的直和, 故可设

$$
d_{1}=\left(f_{1}, f_{2}, \cdots, f_{t}\right): P_{21} \oplus \cdots \oplus P_{2 t} \rightarrow P_{1},
$$

由于每个 $f_{i}$ 都经过 $\operatorname{Ker} d_{0}$ 分解, 且 $\operatorname{Ker} d_{0}$ 不是投射模, 所以每个 $f_{i}$ 均为非同构, 又由极小投射预解式知 $f_{i} \neq 0$ 对任意 $i$, 且 $\operatorname{End}_{\Lambda} P_{1}$ 是域, 所以每个 $P_{2 j}(1 \leqslant j \leqslant t)$ 都不与 $P_{1}$ 同构. 否则, 若 $P_{2 j} \simeq P_{1}$, 则由 $f_{j} \neq 0, f_{j}$ 必为同构.

取 $M=P_{1}, N=\operatorname{Ker}_{1}, M^{\prime}=S_{1}, N^{\prime}=P_{2}$, 令 $\alpha=[M], \beta=[N], \alpha^{\prime}=$ $\left[M^{\prime}\right], \beta^{\prime}=\left[N^{\prime}\right]$, 我们断言不存在 $\lambda \in \mathcal{P}$, 使得 $g_{\alpha \beta}^{\lambda} g_{\alpha^{\prime} \beta^{\prime}}^{\lambda} \neq 0$. 若不然, 设有正合序 列 (6) 和 (7), 因 $M=P_{1}$ 为投射模, 故序列 (6) 可裂, $E \simeq P_{1} \oplus N$, 由序列 (7) 得 到正合列

$$
0 \rightarrow P_{2} \rightarrow P_{1} \oplus N \rightarrow S_{1} \rightarrow 0,
$$

由于每个 $P_{2 j}$ 都不与 $P_{1}$ 同构, 而 $P_{1}$ 是 $S_{1}$ 的投射盖, 所以 $\operatorname{Hom}_{\Lambda}\left(P_{2}, S_{1}\right)=0$, 由 引理 2.6, 或者

$$
P_{2} \simeq P_{1} \oplus N_{1}
$$


$N_{1}$ 是 $N$ 的子模，或者

$$
P_{2} \simeq P_{1}^{\prime} \oplus N
$$

$P_{1}^{\prime}$ 是 $P_{1}$ 的子模, 由 (9) 式得出, 存在 $P_{2 j}$ 与 $P_{1}$ 同构, 矛盾, 由 (10) 式得出 $N$ 是 投射模，则 (8) 式是 $S_{1}$ 的投射预解式，与 $S_{1}$ 的投射维数不小于 2 矛盾.

综上所述, 对于给定的 4 个 $\Lambda$ - 模 $M, N, M^{\prime}, N^{\prime}$, Green 公式左端等于 0 , 以下 说明其 Green 公式的右端非零. 为此只要说明其中有一项非零即可.

考虑 $M$ 的子模 $F=\operatorname{Ker} d_{0}$, 则有正合列

$$
0 \rightarrow F \rightarrow M \rightarrow M^{\prime} \rightarrow 0,0 \rightarrow N \rightarrow N^{\prime} \rightarrow F \rightarrow 0 .
$$

令 $\rho=\left[M^{\prime}\right], \sigma=[F], \sigma^{\prime}=0, \tau=[N]$, 易验证所在 Green 公式右端中对应的项非 零.

设 $F: \bmod \Lambda \rightarrow \bmod \Lambda^{\prime}$ 是等价函子, $M, N, L \in \bmod \Lambda$, 令 $\alpha=[M], \beta=$ $[N], \lambda=[L]$, 在 $\bmod \Lambda^{\prime}$ 中, 令 $F \alpha=[F M], F \beta=[F N], F \lambda=[F L]$, 容易知道 $a_{\alpha}=a_{F \alpha}, g_{\alpha \beta}^{\lambda}=g_{F \alpha F \beta}^{F \lambda}$, 从而 Green 公式在 $\bmod \Lambda$ 中普遍成立当且仅当 Green 公 式在 $\bmod \Lambda^{\prime}$ 中普遍成立, 因此有以下最终定理:

定理 3.3 设 $\Lambda$ 是有限域 $k$ 上带单位元的有限维代数, 则 Green 公式在 $\bmod \Lambda$ 中普遍成立当且仅当 $\Lambda$ 是遗传代数.

证由定理 3.1 和 3.2 以及上述说明知.

致谢 本文所考虑的问题是由肖杰教授向作者提出的，本文的写作也在其 指导下完成，作者在此表示感谢.

\section{参考文献}

1 Green J A. Hall algebras, hereditary algebras and quantum group. Invent Math, 1995, 120: 361 377

2 Ringel C M. Green's theorem on Hall algebras. In: Representations of Algebras and Related Topics. CMS Conference Proceedings. vol 19. American Mathematical Society, 1996. 185 245

3 Xiao J. Drinfeld double and Ringel-Green theory of Hall algebras. J Algebra, 1997, 190: 100 144

4 Sevenhant B, van den Bergh M. A relation between a conjecture of Kac and the structure of the Hall algebra. J Pure Appl Algebra, 2001, 160: 319 332 